\title{
The Brocard and Tucker Circles of a Cyclic Quadrilateral.
}

\author{
By Frederick G. W. Brown.
}

(Received 4th September 1917. Read 9th November 1917.)

1. The application of the geometrical properties of the Brocard and Tucker circles of a triangle to a quadrilateral appears never to have been adequately worked out, as far as the author can discover. Hence, the object of this paper.

Some of the problems involved have been published, under the author's name, as independent questions for solution, and where, in the author's opinion, solutions other than his own have seemed more satisfuctory for the logical treatment of the subject, these solutions have been employed, with due acknowledgments to their authors.

\section{Condition for Brocard points.}

We shall first establish the condition necessary for the existence of Brocard points within a quadrilateral.

Let $A B C D$ (Fig. 1) be a quadrilateral in which a point $X$ can be found such that the $\angle{ }^{B} X A D, X B A, X C B, X D C$ are all equal; denote each of these angles by $\omega$; the sides $B C, C D, D A, A B$ by $a, b, c, d$; the diagonals $B D, A C$ by $e, f$, and the area by $Q$; then

$$
\angle A X B=\pi-\omega-(A-\omega)=\pi-A \text {. }
$$

Similarly $\angle B X C=\pi-B, \angle C X D=\pi-C, \angle D X A=\pi-D$.

Now $A X: \sin \omega=A B: \sin A X B=d: \sin (\pi-A)=d: \sin A$

and $A X: \sin (D-\omega)=c: \sin (\pi-D)=c: \sin D$,

hence, eliminating $A X$ by division

$$
\sin (D-\omega): \sin \omega=d \sin D: c \sin A,
$$




$$
\text { from which } \left.\quad \begin{array}{rl}
\cot \omega & =\frac{d}{c} \operatorname{cosec} A+\cot D . \\
\text { Similarly } \quad \cot \omega & =\frac{c}{b} \operatorname{cosec} D+\cot C, \\
& =\frac{b}{a} \operatorname{cosec} C+\cot b, \\
& =\frac{a}{d} \operatorname{cosec} B+\cot A,
\end{array}\right\}
$$

$\therefore \frac{d}{c} \operatorname{cosec} A+\cot D+\frac{b}{a} \operatorname{cosec} C+\cot B$ $=\frac{c}{b} \operatorname{cosec} D+\cot C+\frac{a}{d} \operatorname{cosec} B+\cot A=2 \cot \omega$.

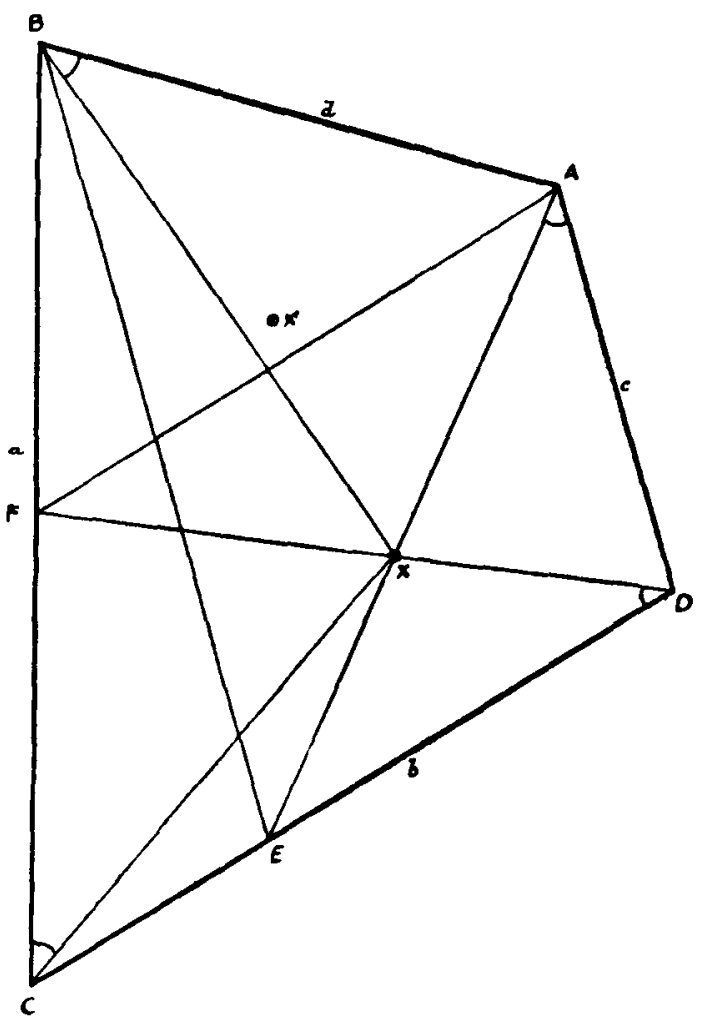

Fig. 1. 


\section{Hence,}

$$
\begin{aligned}
\frac{d}{c} \operatorname{cosec} A-\frac{a}{d} \operatorname{cosec} B+\frac{b}{a} \operatorname{cosec} C-\frac{c}{b} & \operatorname{cosec} D=\cot A-\cot B \\
& +\cot C-\cot D \ldots \ldots \ldots \ldots
\end{aligned}
$$

This is the general condition for all quadrilaterals in its most general form.*

\section{Reduction of Condition for any quadrilateral.}

Condition (2) involves the eight parts of the quadrilateral, but for given data, it will need reduction in order to express it in terms of the data. To effect this it is simpler to start with equations (1). Let us suppose, for example, that the four sides and the angle $C$ are given, and the figure is subject to the limitation that it must be convex, then, since

and

$$
\begin{aligned}
& 2 c d \cos A=c^{2}+d^{2}-e^{2} \\
& e^{2}=a^{2}+b^{2}-2 a b \cos C,
\end{aligned}
$$

$\cos A$ and therefore $\angle A$ become known, there being only one value of $\cos A$ in the eliminant of $e^{2}$ since $A<\pi$.

Put $\operatorname{cosec} A=\alpha, \operatorname{cosec} C=\beta, \operatorname{cosec} B=x, \operatorname{cosec} D=y$ and $\cot \omega=k$, then $\alpha, \beta$ are known and $x, y, k$ unknown constants. Equations (1) now become:-

$$
\begin{aligned}
& k=\frac{d}{c} \cdot a+\sqrt{y^{2}-1}, \\
& k=\frac{c}{b} \cdot y+\sqrt{\beta^{2}-1}, \\
& k=\frac{b}{a} \cdot \beta+\sqrt{x^{2}-1}, \\
& k=\frac{a}{d} \cdot x+\sqrt{\alpha^{2}-1},
\end{aligned}
$$

The condition for Brocard points in terms of $a, b, c, d$ and $\angle C$ is therefore the eliminant of $x, y, k$ in the above equations.

*Vide the author's note in Mathematical Gazette, Vol. IX., pp. 83-85. 
The following method of elimination is due to Lt.Col. Allan Cunningham, R.E.*

For shortness write $E=\sqrt{\beta^{2}-1}, \quad F=\sqrt{\alpha^{2}-1}$, then the equations may be written

$$
\begin{aligned}
\text { (i) } k-\frac{d}{c} \cdot a & =\sqrt{y^{2}-1} \\
\text { (ii) }(k-E) \cdot \frac{b}{c} & =y \\
\text { (iii) } k-\frac{b}{a} \cdot \beta & =\sqrt{x^{2}-1} \\
\text { (iv) }(k-F) \frac{d}{a} & =x
\end{aligned}
$$

Squaring and taking the differences of (i) and (ii), and of (iii) and (iv), we get the two following equations independent of $x$ and $y$ :-

$$
(k-E)^{2} \cdot \frac{b^{2}}{c^{2}}-\left(k-\frac{d}{c} \alpha\right)^{2}=1 ; \quad(k-k)^{2} \cdot \frac{d^{2}}{a^{2}}-\left(k-\frac{b}{a} \cdot \beta\right)^{2}=1,
$$

which may be written as quadratics in $k$, thus

or

$$
\begin{gathered}
k^{2}\left(b^{2}-c^{2}\right)+2 k\left(c d \alpha-b^{2} E\right)+\left(b^{2} E^{2}-d^{2} \alpha^{2}-c^{2}\right)=0, \\
k^{2}\left(d^{2}-a^{2}\right)+2 k\left(a b \beta-d^{2} F^{\prime}\right)+\left(d^{2} F^{2}-b^{2} \beta^{2}-a^{2}\right)=0, \\
p k^{2}+2 q k+r=0, \\
p^{\prime} k^{2}+2 q^{\prime} k+r^{\prime}=0,
\end{gathered}
$$

the eliminant of which is

$$
\left(p r^{\prime}-p^{\prime} r\right)^{2}+4\left(p^{\prime} q-p q^{\prime}\right)\left(q r^{\prime}-q^{\prime} r\right)=0,
$$

which is therefore the required condition.

When the substitutions are made, however, the relation is so cumbersome that it is practically of very little value.

4. Reduction of Condition for a cyclic quadrilateral.

. When the quadrilateral is inscribed in a circle, the condition for Brocard points becomes very simple, and many interesting analogies to the triangle are revealed.

Going back to (2), we have for a cyclic figure, $\operatorname{cosec} A=\operatorname{cosec} C, \operatorname{cosec} B=\operatorname{cosec} D, \cot A=-\cot C, \cot B=-\cot D$, since

$$
-A+\angle C=\angle B+\angle D=\pi \text {, }
$$

- Mathematical Questions and Solutions (F. Hodgson, London), Vol. 2, p. 47. 
hence, (:2) becomes

$$
\left(\frac{d}{a}+\frac{b}{a}\right) \operatorname{cosec} A-\left(\frac{a}{d}+\frac{c}{b}\right) \operatorname{cosec} B=0
$$

or

$$
b d(a d+b c) \sin H=a c(a b+c d) \sin A .
$$

But

$$
\underline{2} Q=(a b+c d) \sin A=(a d+b c) \sin B .
$$

Hence,

$$
a c=b d,
$$

and from Ptolemy's theorem, $e f=a c+b d$.

$$
\therefore \quad a c=b d=\frac{1}{2} e f
$$

the required condition.

We shall therefore confine the following investigation to a cyclic quadrilateral.

\section{Geometrical Proof of' (4).}

This simple relation for Brocard points may also be established geometrically, and the following proof is based on one given by Mr W. F. Beard, M.A.*

Produce $A X, D X$ (Fig. 1) to meet $D C, B C$ in $E$, $F$ respectively, join $E B, F A$, then

$$
\begin{aligned}
& \angle B X A=\pi-A=\angle C \\
& \angle X B A=\angle F D C \text {. } \\
& \therefore \triangle \mathrm{s} B A X, F D C \text { are similar, } \\
& \therefore \quad C D: D F=B X: A B \\
& \text { or } A B \cdot C D=D H^{\top} . B X \text {. } \\
& \text { Again, } \quad-A X F=\text { supplement of } \angle A X D \\
& \text { = supplement of } \pi-D=\angle D \\
& \text { = supplement of } \angle B \text {. } \\
& \therefore \quad A, B, F, X \text { lie on a circle, } \\
& \angle B F A=\angle B X A=\pi-A=\angle C \text {. } \\
& \therefore A F \text { is parallel to } D C \text {. } \\
& \angle D A X=\angle B C X \\
& \angle B X C=\pi-B=\angle D \text {. }
\end{aligned}
$$

hence,

Similariy

Now

* Mathematical Questions and Solutions (F, Hodgson, London), Vol. 3, pp. 2-3. 
and

$$
A D: A E=X C: B C \text {. }
$$

$$
\therefore \quad A D . B C=A E . X C \text {. }
$$

Finally, $\angle B X C=\angle D$ and $\angle A X F=\angle D$, already proved.

$$
\therefore \angle B X C=\angle A X F=\angle E X D
$$

and $\angle B C X=\angle E D X=\angle D F A, \quad \because A F \| D E$. $\therefore \triangle \mathrm{s} B X C, A F X, D X E$ are similar;

hence, $\quad B X: X C=E X: X D=A X: X F=A E: D F$.

hence, $A B . C D=A D . B C$.

\section{Second Brocard Point.}

The point $X$ has a corresponding one $X^{\prime}$ such that the angles $X^{\prime} A B, X^{\prime} B C, X^{\prime} C D, X^{\prime} D A$ are all equal, and it may be shown in a precisely similar manner as for $X$ that the necessary condition for its existence is $a c=b d=\frac{1}{2} e f$.

Hence, when this condition is fulfilled, there are two Brocard points, just as in the case of a triangle.

Let $\omega^{\prime}=$ each of the equal angles $X^{\prime} A B$, etc., then, as in Art. 2, it may be shown that

$$
\cot \omega^{\prime}=\frac{c}{d} \cdot \operatorname{cosec} A+\cot B
$$

But $\operatorname{cosec} A=\operatorname{cosec} C$, and from (4)

$$
\begin{gathered}
c: d=b: a . \\
\therefore \quad \cot \omega^{\prime}=\frac{b}{a} \operatorname{cosec} C+\cot B=\cot \omega, \text { from }(1) . \\
\therefore \quad \omega^{\prime}=\omega .
\end{gathered}
$$

$X$ and $X^{\prime}$ are therefore isogonal conjugates.

7. Geometrical Construction for a Cyclic Quadrilateral having Brocard Points.

A cyclic quadrilateral $A B C D$, such that $A B . C D=A D . B C$, may be constructed by the following general method.

Draw any straight line $L L_{1}$ (Fig. 2); take any point $A$ in it and mark off $A L_{2}=A L$. Through $A$ draw any straight line $Q Q_{1}$; join $Q L Q L_{2}$, and draw $Q_{1} L_{1}$ antiparallel to $L Q$ with respect to $\angle Q_{1} A L_{1}$; bence, draw $B D$ parallel to $Q_{1} L_{1}$ and equal to $Q L_{2}$.

Describe the circumcircle to the $\triangle A B D$ and in it place a chord $B C$ such that $\angle D B C=\angle A L_{2} Q$; join $C D$, then $A B C D$ is the required quadrilateral. 
Since $A, B, C, D$ are concjclic points,

$$
\begin{aligned}
& \therefore \angle B C D=\text { supplement of } \angle B A D=\angle L_{2} A Q \\
& \text { and } \left.\begin{array}{rl}
-D B C & =\angle A L_{2} Q \\
B D & =Q L_{2}
\end{array}\right\} \text { By construction. } \\
& \therefore \triangle \mathrm{s} B C D, Q A L_{\mathrm{g}} \text { are congruous. } \\
& \text { Hence, } B C=A L_{2} \text { and } C D=A Q \text {. }
\end{aligned}
$$



Fig. 2.

Again, since $B D, L Q$ are antiparallels with respect to $\angle B A D$, $\therefore \quad L, Q, D, B$ are concyclic points

$$
\therefore \quad L A . A D=Q A . A B \text {. }
$$

But

$$
L A=A L_{2}=B C \text { and } Q A=C D, \text { already proved. }
$$$$
\therefore \quad B C \cdot A D=C D, A B \text {. }
$$ 
This general construction may, with a little modification, be adapted to most cases of particular given conditions.

\section{Location of Brocard Points.}

As in the case of the triangle, $X, X^{\prime}$ may be located by Milne's construction, i.e., by describing circles on $A B, B C, C D, D A$ touching the sides $A D, A B, B C, C D$ respectively; $X$ is then their common point of intersection. Similarly if the circles described on $A B$, $B C, C D, D A$ touch $B C, C D, D A, A B$ respectively, then $X^{\prime}$ is their common point of intersection.

The geometrical proof given in Art. 5 affords, however, a much simpler construction, for when $A X, D X$ are produced to cut $C D, B C$ in $E, F$ respectively (Fig. 1), then $B E, A F$ are parallel respectively to $A D, C D$, hence, the following construction:

Draw from two consecutive angular points, $A, B, A F, B E$ parallel to $C D, A D$ respectively to cut $B C$ in $F$ and $C D$ in $E$; join $E A, F D$ and their point of intersection will be $X$.

Similarly by drawing from $C, D$ lines parallel to $A D, A B$ respectively to cut $A B$ in $E^{\prime}$ and $B C$ in $F^{\prime}$, then $X^{\prime}$ is the point of intersection of $E^{\prime} D, F^{\prime} A$.

\section{Important Formulae.}

The expressions $a d+b c, a b+c d, a c+b d$ are of such frequent occurrence in connection with the cyclic quadrilateral that it will facilitate the discussion if we give the forms they may assume when condition (4) is fulfilled.

$$
a d+b c=\frac{a c d+b c^{2}}{c}=\frac{b d^{2}+b c^{2}}{c}=\frac{b}{c}\left(c^{2}+d^{2}\right),
$$

also

$$
\left.\begin{array}{rl}
a d+b c= & \frac{a b d+b^{2} c}{b}=\frac{a^{2} c+b^{2} c}{b}=\frac{c}{b}\left(a^{2}+b^{2}\right) . \\
\therefore \quad a d+b c=\frac{b}{c}\left(c^{2}+d^{2}\right) & =\frac{c}{b}\left(a^{2}+b^{2}\right) \\
a b+c d & =\frac{c}{d}\left(a^{2}+d^{2}\right)=\frac{d}{c}\left(b^{2}+c^{2}\right) \\
\text { (4) } \quad a c+b d & =2 a c=2 b d=e f
\end{array}\right\}
$$

Similarly

and from (4)

The following formulae are now given for reference in their usual forms. Modification by (5) will be made as necessary. 
Let $R=$ radius of circumcircle of quadrilateral, then

$$
\left.\begin{array}{l}
\sin A=\sin C=\frac{e}{2 R} \\
\sin B=\sin D=\frac{f}{2 R}
\end{array}\right),
$$

and since $\quad 2 Q=(a b+c d) \sin A=(a d+b c) \sin B$,

$$
\therefore \quad 4 R Q=e(a b+c d)=f(a d+b c) .
$$

Also

$$
\left.\begin{array}{rl}
e^{2}(a b+c d) & =(a c+b d)(a d+b c)=2 a c(a d+b c) \\
f^{2}(a d+b c) & =(a c+b d)(a b+c d)=2 a c(a b+c d)
\end{array}\right\} .
$$

Again, from (1),

$$
\begin{aligned}
2 \cot \omega & =\frac{d}{c} \operatorname{cosec} A+\cot D+\frac{b}{a} \operatorname{cosec} C+\cot B \\
& =\frac{a d+b c}{a c} \cdot \operatorname{cosec} A \\
& =\frac{a b+c d}{b d} \operatorname{cosec} B, \text { similarly. }
\end{aligned}
$$

$\therefore \quad 2 a c \cot \omega=(a d+b c) \operatorname{cosec} A=(a b+c d) \operatorname{cosec} B$.

Finally, from (6) and (7),

$$
\begin{aligned}
& 2 a c \cot \omega=\frac{4 R Q}{f} \cdot \frac{2 R}{e}=\frac{8 R^{2} Q}{2 a c}, \text { from (4), } \\
& \therefore \quad \cot \omega=\frac{2 R^{2} Q}{a^{2} c^{2}} . \ldots \ldots \ldots \ldots \ldots \ldots \ldots \ldots \ldots \ldots \ldots \ldots \ldots \ldots \ldots
\end{aligned}
$$

Other expressions for the functions of $\omega$ will be found in Art. 12.

10. Distances from angular points and coordinates of $X, X^{\prime}$. From the triangle $A X B$ (Fig. 3),

$X A: \sin \omega=A B: \sin A X B=d: \sin A=2 R d: e$, from (6).

$\therefore \quad X A=\frac{2 R d}{e} \cdot \sin \omega ; \operatorname{similarly}, X^{\prime} A=\frac{2 R c}{e} \cdot \sin \omega$,

and $X B=\frac{2 R a}{f} \cdot \sin \omega ; \quad, \quad X^{\prime} B=\frac{2 R d}{f} \cdot \sin \omega$,

$$
\begin{array}{lll}
X C=\frac{2 R b}{e} \cdot \sin \omega ; & , & X^{\prime} C=\frac{2 R a}{e} \cdot \sin \omega, \\
X D=\frac{2 R c}{f} \cdot \sin \omega ; & & X^{\prime} D=\frac{2 R b}{f} \cdot \sin \omega
\end{array}
$$


From these values the following important geometrical results are readily deduced :

(a) $X A \cdot X B \cdot X C^{\prime} \cdot X D=X^{\prime} A \cdot X^{\prime} B \cdot X^{\prime} C^{\prime} \cdot X^{\prime} D=4 R^{4} \sin ^{6} \omega$

(b) $X A \cdot X^{\prime} D=X B \cdot X^{\prime} A=X C^{\prime} \cdot X^{\prime} B=X D \cdot X^{\prime} C=2 R^{\prime \prime} \sin ^{2} \omega$

(c) $\frac{X A \cdot X C}{X B \cdot X D}=\frac{X^{\prime} A \cdot X^{\prime} C}{X^{\prime} B \cdot X^{\prime} D}=\frac{f^{2}}{e^{\underline{2}}}=\frac{A C^{2}}{B D^{2}}=\frac{(a b+c d)^{2}}{(a d+b c)^{2}}$

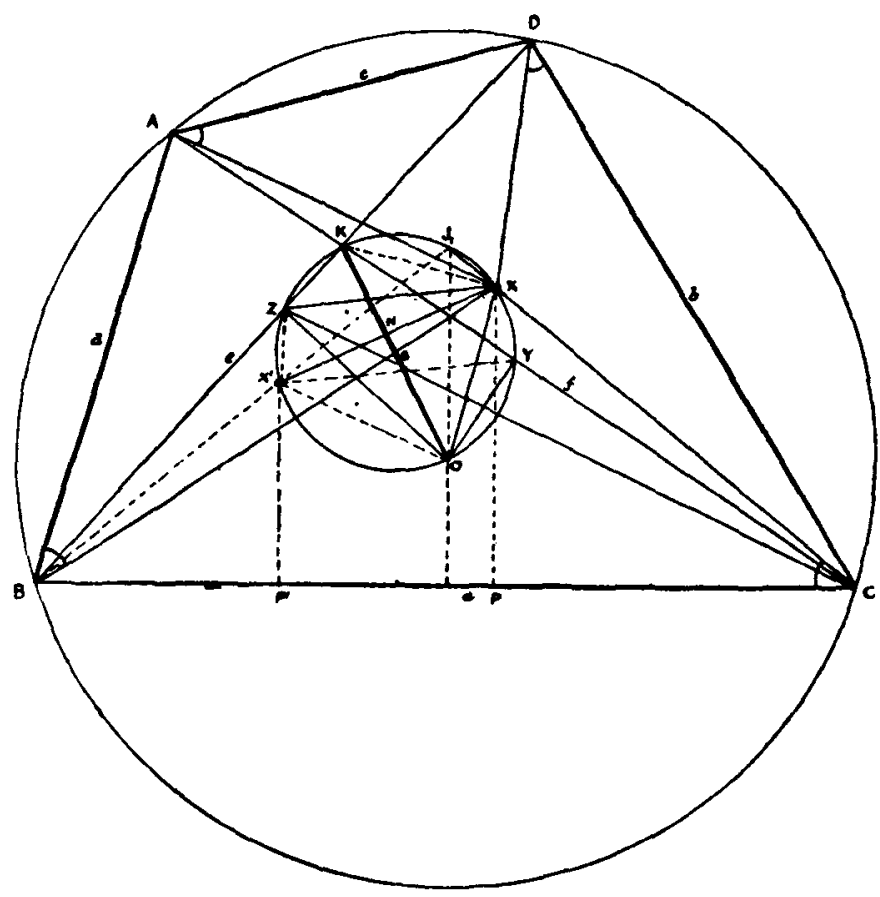

Fig. 3.

To determine the Cartesian co-ordinates of $X, X^{\prime}$ with reference to any of the sides, say $B C$, let $P, P^{\prime}$ be the feet of the perpendiculars from $X, X^{\prime}$ to $B C$, then 


$$
\left.\begin{array}{rl}
C P=C X \cos \omega & =\frac{2 R b}{e} \cdot \sin \omega \cdot \cos \omega, \text { from }(11), \\
& =\frac{R b}{e} \sin 2 \omega \\
P X=C X \sin \omega & =\frac{2 R b}{e} \sin ^{2} \omega \\
B P^{\prime} & =\frac{R d}{f} \cdot \sin 2 \omega \\
P^{\prime} X^{\prime} & =\frac{2 R d}{f} \cdot \sin ^{2} \omega
\end{array}\right\} \ldots \ldots \ldots \ldots \ldots
$$

Similarly,

Like expressions may similarly be found with reference to each of the other sides.

From (13) and (4) we have

$$
B P^{\prime} . C P=\frac{1}{2} R^{2} \sin ^{2} 2 \omega \text { and } P X . P^{\prime} X^{\prime}=2 R^{2} \sin ^{4} \omega ;
$$

these rectangles, being thus constant, are therefore the same for every side.

To find the distance between $X$ and $X^{\prime}$, we have

$$
\begin{aligned}
X X^{\prime 2} & =\left(B P-B P^{\prime}\right)^{2}+\left(P X-P^{\prime} X^{\prime}\right)^{2} \\
& =B X^{2}+B X^{\prime 2}-2(a-C P) \cdot B P^{\prime}-2 P X . P^{\prime} X^{\prime} \\
& =4 R^{2} \sin ^{2} \omega\left[\frac{a^{2}+d^{2}}{f^{2}}-\frac{a d}{f R} \cot \omega+\cos ^{2} \omega-\sin ^{2} \omega\right] \\
& =4 R^{2} \sin ^{2} \omega \cdot \cos 2 \omega, \quad \text { from }(6) \text { and }(9) .
\end{aligned}
$$

$\therefore X \Gamma^{\prime}=2 R \sin \omega \cdot \cos ^{\frac{1}{2}} 2 \omega$.

\section{The Brocard Circle.}

Let $O$ be the circumcentre, $K$ the intersection of the diagonals $A C, B D$, and $Y, Z$ their respective mid-points (Fig. 3). Join

$$
O K, O Y, O Z, O X, O X^{\prime}, X Y, X Z, Z K, X^{\prime} Y, X^{\prime} Z \text {. }
$$

Then, since

$$
\angle K Y O=\angle O Z K=\frac{1}{2} \pi
$$

$$
\therefore \quad O, Y, K, Z \text {, are concyclic. }
$$

Again,

$$
\angle Z B C=\angle C A D \text {, and from (4), }
$$

$$
a: \frac{1}{2} e=f: c \text {, }
$$

or $B C: B Z=A C: A D$.

$\therefore \triangle \mathrm{s} Z B C, A C D$ are similar;

hence,

$$
\angle B Z C=\angle A D C=\angle D=\angle B X C \text {. }
$$

$\therefore \quad Z, X, C, B$, are concyclic points. 
Similarly, it may be shown that $X Y^{\prime} C^{\prime} D, X^{\prime} Y B C, X^{\prime} Z D C^{\prime}$ are each cyclic.

Now, because $Z X C B$ is cyclic,

$$
\therefore \quad-K Z X=\text { supplement of }-B Z X=-B C X=\omega \text {. }
$$

Because $X Y C D$ is cyclic,

$\angle K Y X=$ supplement of $-X Y C=-X D C=\omega$.

$$
\therefore \quad-K Z X=-K Y X:
$$

hence,

$$
X, K, Z, Y \text { are concyclic. }
$$

Similarly

$$
-K Y X^{\prime} \text { and }-K Z X^{\prime}=\pi-\omega \text {, }
$$

therefore

$$
X^{\prime}, K, Z, Y \text { are concyclic, }
$$

hence, the six points $O, Y, X, K, Z, X^{\prime}$ lie on a circle whose diameter is $O K$; this is the Brocard Circle of the quadrilateral.

Let $C X, B X^{\prime}$ intersect at $I_{1}$, then because

$$
\begin{gathered}
\therefore J_{1} C B=\angle J_{1} B C=\omega, \\
\therefore \quad J_{1} B=J_{1} C,
\end{gathered}
$$

and $J_{1}$ lies on the perpendicular to $B C^{r}$ at its mid-point,

$\therefore \quad J_{1} O$ produced intersects $B C$ at right angles at its mid-point.

$$
\begin{gathered}
\therefore \quad \angle O J_{1} X=\angle O J_{1} X^{\prime}=\frac{1}{2} \pi-\omega, \\
\therefore \quad O J_{1} \text { bisects } \angle X^{\prime} J_{1} X,
\end{gathered}
$$

and because $\angle X Y O=\angle O Y K+\angle K Y X=\frac{1}{2} \pi+\omega$,

$$
\therefore \quad \angle \mathrm{s} O J_{1} X, X Y O \text { are supplementary, }
$$

$\therefore J_{1}$ lies on the Brocard circle.

Similarly if $J_{2}$ be the intersection of $D X, C X^{\prime}, J_{3}$ that of $A X, D X^{\prime}$, and $J_{4}$ that of $B X, A X^{\prime}$, it may be shown that $J_{2}, J_{3}, J_{\downarrow}$ lie on the Brocard circle.

Hence the Brocard circle passes through the following ten points: the two Brocard points $\left(X, X^{\prime}\right)$, the mid-points of the diagonals $(Y, Z)$, the intersection of the diagonals $(K)$, the circumcentre $(O)$,

the intersections of the joins of the Brocard points and the angular points of the quadrilateral, i.e., the apices of the isosceles triangles having the sides as their respective bases and $\omega$ as the equal angles. 
12. Functions of $\omega$ and its maximum value.

Before proceeding to find the Brocard radius it is necessary to evaluate some of the functions of $\omega$.

Let $u_{t}, u_{b}, u_{c}, u_{c l}$ be the lengths of the perpendiculars from $K$ to the sides $B C, C D, D A, A B$ respectively; then, since $O K$ is the diameter of the Brocard circle,

$$
\begin{aligned}
& \therefore \quad K K J_{1} O=\frac{1}{2} \pi . \\
& \therefore \quad K J_{1} \text { is parallel to } B C .
\end{aligned}
$$

$\therefore \quad u_{u}=$ perpendicular from $J_{1}$ to $B C=\frac{1}{2} a \cdot \tan \omega$.

Similarly, $u_{b}=\frac{1}{2} b \cdot \tan \omega, \quad u_{c}=\frac{1}{2} c \cdot \tan \omega, \quad u_{d}=\frac{1}{2} d \cdot \tan \omega$.

Now the area of the quadrilateral

$$
\begin{aligned}
Q & =\triangle A K B+\triangle B K C+\triangle C K D+\triangle D K A \\
& =\frac{1}{2} a u_{a}+\frac{1}{2} b u_{b}+\frac{1}{2} c u_{c}+\frac{1}{2} d u_{d} \\
& =\frac{1}{4}\left(a^{2}+b^{2}+c^{2}+d^{2}\right) \cdot \tan \omega . \\
& \therefore \quad \cot \omega=\frac{\searrow a^{2}}{4 Q} \cdot \ldots \ldots \ldots \ldots \ldots \ldots \ldots \ldots \ldots \ldots \ldots \ldots \ldots
\end{aligned}
$$

But $\operatorname{cosec}^{2} \omega=1+\cot ^{2} \omega=\frac{16 Q^{2}+\left(\dot{\Sigma} a^{2}\right)^{2}}{16 Q^{2}}$

$$
\left.\begin{array}{l}
=\frac{\searrow a^{2} b^{2}+2 a b c d}{4 Q^{2}} \\
=\frac{(a b+c d)^{2}+(a d+b c)^{2}}{4 Q^{2}} \text { by }(t) \\
=\operatorname{cosec}^{2} A+\operatorname{cosec}^{2} B
\end{array}\right\} \ldots
$$

Hence

$$
\cot ^{n} \omega=1+\cot ^{\prime \prime} A+\cot ^{2} B \text {. }
$$

Again, $\quad \cos ^{2} \omega=\cot ^{2} \omega \cdot \sin ^{2} \omega=\frac{1}{4} \cdot \frac{\left(2 a^{2}\right)^{2}}{\sum a^{2} b^{2}+2 a^{2} c^{2}}$

since by (4), $a b c d=a^{2} c^{2}=b^{2} d^{2}$

$$
\left.\begin{array}{rl}
\sin ^{2} 2 \omega=4 \sin ^{2} \omega \cdot \cos ^{2} \omega & =\frac{4\left(\Sigma a^{2}\right)^{2} \cdot Q^{2}}{\left(\Sigma a^{2} b^{2}+2 a^{2} c^{2}\right)^{2}} \\
\therefore \quad \sin 2 \omega=\frac{2 \cdot \Sigma a^{2} \cdot Q}{\Sigma a^{2} b^{2}+2 a^{2} c^{2}} & \ldots \ldots \\
\cos 2 \omega & =2 \cos ^{2} \omega-1 \\
& =\frac{\sum a^{4}-4 a^{2} c^{2}}{2\left(\Sigma a^{2} b^{2}+2 a^{2} c^{2}\right)} \\
& =\frac{1}{2} \cdot \frac{\left(a^{2}-c^{2}\right)^{2}+\left(b^{2}-d^{2}\right)^{2}}{(a b+c d)^{2}+(a d+b c)^{2}}
\end{array}\right\} .
$$

$6 \mathrm{Vol} .36$ 
From (15b) we have

$$
\operatorname{cosec} \omega=\left(\operatorname{cosec}^{2} A+\operatorname{cosec}^{2} B\right)^{\frac{1}{2}},
$$

and since the right-hand side is the sum of two squares, it is always positive, hence $\operatorname{cosec} \omega$, and therefore $\omega$, is always real.

Now the minimum value of either $\operatorname{cosec} A$ or $\operatorname{cosec} B$ is 1 , hence $\operatorname{cosec} \omega$ is not less than $\sqrt{2}$, i.e., $\omega$ cannot exceed $\frac{\pi}{4}$ or $45^{\circ}$.

\section{Radius of the Brocard Circle.}

From Art. 11 we have

$$
\begin{gathered}
\angle K Z X=\angle K Y X=-\kappa Y X^{\prime}=\omega \quad \text { (Fig. 3). } \\
\therefore K Y \text { bisects } \angle X Y X^{\prime} .
\end{gathered}
$$

But $\angle K Y X=-K O X$ in the same segment,

and

$$
\begin{aligned}
& \angle K Y X^{\prime}=\angle K O X^{\prime} \quad, \quad " \quad " \\
& \angle K O X=-K O X^{\prime}=\omega,
\end{aligned}
$$

and

$$
O K \text { bisects } \angle X O X^{\prime} \text {; }
$$

hence, since $O K$ is a diameter,

$$
\therefore \quad O X=O X^{\prime}, \quad K X=K X^{\prime}
$$

and $X X^{\prime}$ is perpendicular to $O K$.

Let $X X^{\prime}$ intersect $O K$ at $N$, then

$$
X N=N X^{\prime}=\frac{1}{2} X X^{\prime}=R \sin \omega \cdot \cos ^{\frac{1}{2}} 2 \omega, \text { from (14). }
$$

But from above,

$$
\begin{aligned}
& \angle X O N=\angle X O K=\omega, \\
& \angle O N X=\angle O X K=\frac{1}{2} \pi . \\
& \therefore \quad \angle K X N=\angle X O N=\omega .
\end{aligned}
$$

Let $\beta=$ radius of Brocard circle, then

$$
\begin{aligned}
& 2 \beta=O K=O N+N K \\
&=X N(\cot \omega+\tan \omega) \\
&=2 R \sin \omega \cdot \cos ^{\frac{1}{2}} 2 \omega \cdot \operatorname{cosec} 2 \omega, \text { from above, } \\
&=R \sec \omega \cdot \cos ^{\frac{1}{2}} 2 \omega \\
& \therefore \quad \beta\left.=\frac{1}{2} R \sec \omega \cdot \cos ^{\frac{1}{2}} 2 \omega\right) \\
&\left.=\frac{R\left(\Sigma a^{4}-4 a^{2} c^{2}\right)^{\frac{1}{2}}}{\sqrt{2} \cdot \Sigma a^{2}}\right\} \ldots \ldots \ldots \ldots \ldots \ldots \ldots \ldots \ldots \ldots \ldots \ldots \ldots \ldots \\
& \text { from }(15 d) \text { and }(15 f) .
\end{aligned}
$$


The distance from each of the Brocard points to the circumcentre may now easily be found, for

$$
\begin{aligned}
O X & =N X \operatorname{cosec} X O N \\
& =R \sin \omega \cdot \cos ^{\frac{1}{3}} 2 \omega \cdot \operatorname{cosec} \omega \\
& =R \cos ^{\frac{1}{2}} 2 \omega . \\
\therefore \quad O X & =O X^{\prime}=R \cos ^{\frac{1}{2}} 2 \omega .
\end{aligned}
$$

Similarly $X K=K X^{\prime}=R \tan \omega \cdot \cos ^{\frac{1}{2}} 2 \omega$.

\section{T'ucker Circles.}

Take any point $A^{\prime}$ (Fig. 4) in $K^{\prime} A$ and through it draw $H A^{\prime} H^{\prime}$ parallel to $A B$ to intersect $B D$ in $B^{\prime}$, and $G A^{\prime} G^{\prime}$ parallel to $A D$ to intersect $B D$ in $D^{\prime}$. Through $D^{\prime}$ draw $F D^{\prime} F^{\prime \prime}$ parallel to $C D$ to intersect $A C^{\prime}$ in $C^{\prime}$; join $B^{\prime} C^{\prime}$ and produce it in both directions to meet $A B, C D$ in $E, E^{\prime}$ respectively, then

$$
\begin{gathered}
K B^{\prime}: B^{\prime} B=K^{\prime} A^{\prime}: A^{\prime} A=K D^{\prime}: D^{\prime} D=K C^{\prime \prime}: C^{\prime} C . \\
\therefore \quad B^{\prime} C^{\prime} \text { is parallel to } B C .
\end{gathered}
$$

Join $I I G^{\prime}, E H^{\prime}, F E^{\prime}, G F^{\prime \prime}$, then

$$
A^{\prime} I I: A^{\prime} A=\sin A^{\prime} A H: \sin A^{\prime} H A=\sin C A D: \sin A^{\prime} H D
$$

$$
=b: 2 R \sin A \text {, }
$$

since $2 R \sin C A D=b$.

Similarly

$$
A^{\prime} G^{\prime}: A^{\prime} A=a: 2 R \sin A .
$$

Hence, by division $A^{\prime} H: A^{\prime}\left(r^{\prime}=b: a\right.$

$$
\begin{aligned}
& =c: d \text { from }(4) \\
& =A D: A B \\
& =A^{\prime} D^{\prime}: A^{\prime} B^{\prime} . \\
\therefore \quad A^{\prime} H . A^{\prime} B^{\prime} & =A^{\prime} G^{\prime} . A^{\prime} D^{\prime} . \\
\therefore \quad B^{\prime}, G^{\prime}, H, D^{\prime} & \text { are concyclic }
\end{aligned}
$$

hence, $G^{\prime} I I$ is antiparallel to $B D$ with respect to $\angle A$,

Similarly $F E^{\prime}, H^{\prime} E, F^{\prime} G$ are antiparallel to $B D$ and $A C$ with respect to $\therefore \mathrm{s} C, B, D$ respectively.

Let $K A, K B, K^{\prime}, K D$ intersect $H G^{\prime}, E H^{\prime} F E^{\prime \prime} G F^{\prime}$ in $T_{1}^{\prime}, T_{2}$, $T_{3}, T_{4}$ respectively, then, since $A G^{\prime} A^{\prime} H$ is a parallelogram,

$$
\therefore \quad T_{1}^{\prime} \text { is the mid-point of } I I G^{\prime} \text {. }
$$

$\therefore K A$ is the symmedian of the $\triangle A B D$ with respect to $\angle A$.

Similarly $K B, K C, K D$ are the symmedians of $\triangle \mathrm{s} A B C, C B D$, $A D C^{\prime}$ with respect to $\angle \mathrm{s} B, C, D$ respectively. 
Again, because $H G^{\prime}$ is an antiparallel to $B D$ and $O$ is the circumcentre of the $\triangle A B D$,

$\therefore O A$ is perpendicular to $H G^{\prime}$.

Draw $T_{1} U$ parallel to $O A$ to meet $O K$ in $U$, then

$U T_{1}$ also is perpendicular to $H G^{\prime}$.

$\therefore \quad U G^{\prime 2}=U T_{1}^{2}+T_{1} G^{\prime 2}=U T_{1}^{2}+T_{1} H^{2}=U H^{2}$.

$\therefore \quad U G^{\prime}=U H$.

Similarly, $\quad U F^{\prime \prime}=U G, U E^{\prime}=U F, U E=U H^{\prime}$.

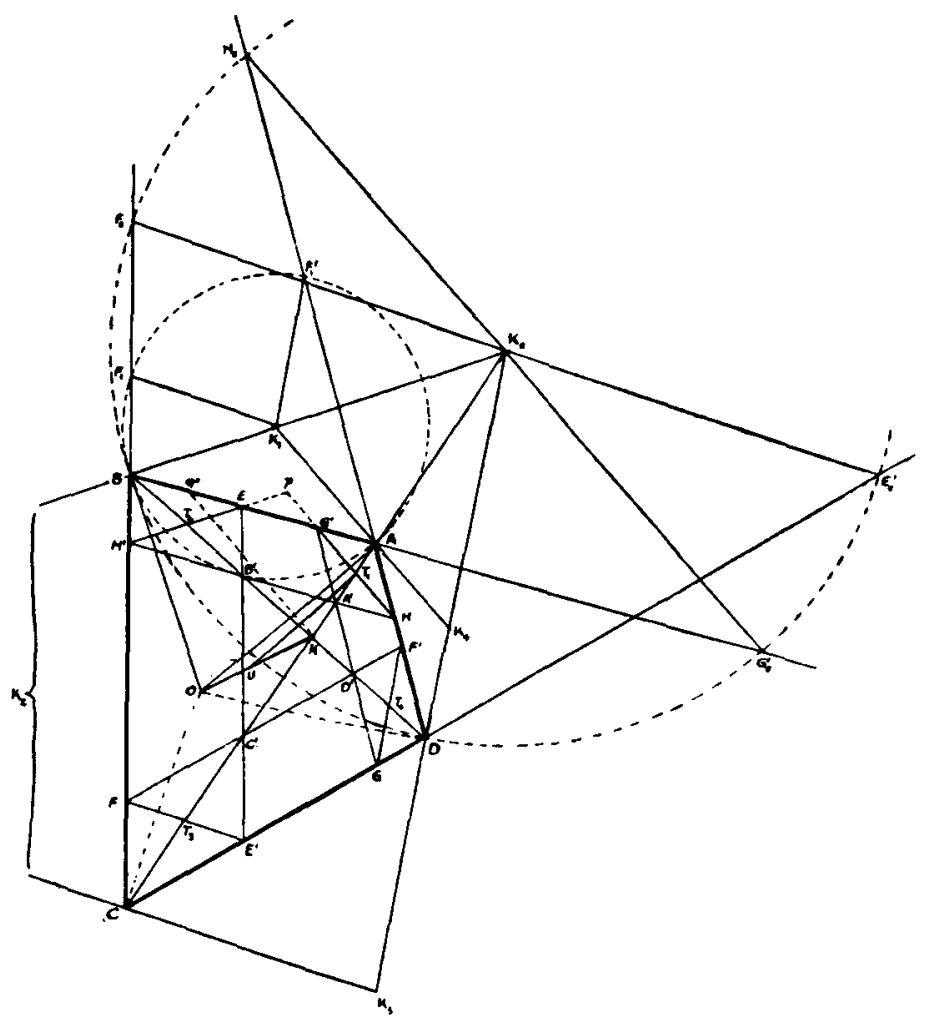

Fig. 4.

Let $H^{\prime} E, H G^{\prime}$ produced intersect in $p$, then $-p H H=\angle H G^{\prime} A=\angle B D A=\angle B C A=-B E H I^{\prime}=-p H I^{\prime} H$.

$$
\therefore p H^{\prime}=p H \text {, }
$$


and since $E G^{\prime}$ is parallel to $H H^{\prime}$,

$$
\therefore p E=p G^{\prime} \text {. }
$$

Hence,

$$
E H^{\prime}=G^{\prime} H \text {. }
$$

Similarly, $E H^{\prime}=E^{\prime} F, E^{\prime} F=F G, F G=H G^{\prime}$.

$$
\therefore \quad H G^{\prime}=E H^{\prime}=F E^{\prime}=G F^{\prime} \text {. }
$$

Further, $\quad U T_{1}: O A=U K: O K=U T_{2}: O B$.

$$
\therefore \quad U T_{1}=U T_{2} \text {. }
$$

Similarly,

$$
U T_{3}=U T_{3}=U T_{4}
$$

Hence $T_{1}, T_{2}, T_{3}, T_{4}$ lie on a circle whose centre is $U$, to which $E^{\prime \prime} F^{\prime}, H^{\prime} E, G^{\prime} H, F^{\prime} G$ are tangents.

Since, however, these tangents have already been proved equal,

$\therefore$ the eight points $E, E^{\prime}, F, F^{\prime}, G, G^{\prime}, H, H^{\prime}$ also lie on a circle whose centre is $U$. This is a Tucker Circle of the quadrilateral.

\section{Radius of a Tucker Circle.}

It is clear that after having once established the geometrical properties of the figure, as in the preceding article, we may take $U$ to be any point in $O K$ and then proceed to determine the radius of the Tucker Circle having $U$ as its centre, by finding the points where it intersects the sides of the quadrilateral. $O K$ is thus the locus of the centres of all the Tucker Circles. To find a general expression for their radii, let

$$
O U: O K=\lambda: 1 \text {, }
$$

and let $\rho=$ radius of the circle whose centre is $K$; then $\rho$ is the semi-length of the antiparallels through $K$.

Now

$U T_{1}: O A=U K: O K$

or

$$
\begin{aligned}
& U T_{1}: R=1-\lambda: 1 . \\
& \therefore U T_{1}=R(1-\lambda) .
\end{aligned}
$$

This is the radius of the circle which passes through $T_{1}, T_{2}, T_{3}^{\prime}, T_{4}$.

Also

$$
\begin{aligned}
G^{\prime} T_{1}: \rho & =A T_{1}^{\prime}: A K \\
& =O U: O K \\
& =\lambda: 1 . \\
\therefore \quad G^{\prime} T_{1}^{\prime} & =\lambda \rho .
\end{aligned}
$$


If therefore $\tau=$ radius of a Tucker Circle,

$$
\begin{aligned}
\tau^{2}=U G^{\prime 2} & =U T_{1}^{2}+T_{1} G^{\prime 2} \\
& =R^{2}(1-\lambda)^{2}+\lambda^{2} \rho^{2} .
\end{aligned}
$$

To completely define $\tau$, however, it is necessary to find a value for the unknown $\rho$.

Now, if the parallel to $H G^{\prime}$ through $K$ meets $A B$ in $G^{\prime \prime}$, then the perpendicular from $K$ to $A B=K G^{\prime \prime} \sin A G^{\prime \prime} K=\rho \sin A D B=\frac{1}{2} \rho \cdot \frac{d}{R}$.

$$
\begin{aligned}
\therefore \quad & 2 R u_{d}=\rho d \\
& \rho=R \tan \omega .
\end{aligned}
$$

or

Putting this value in the above expression for $\tau$, we get

$$
\begin{aligned}
\tau^{2} & =R^{2}(1-\lambda)^{2}+\lambda^{2} R^{2} \tan ^{2} \omega \\
& =R^{2}\left(1-2 \lambda+\lambda^{2} \sec ^{2} \omega\right) .
\end{aligned}
$$

16. Particular cases of Tucker Circles.

When $\lambda=1, U$ is coincident with $K$, and from (19),

$$
\tau=R \tan \omega=\rho .
$$

This is therefore the radius of the Tucker Circle, which in the triangle is known as the Cosine Circle. It may, however, be appropriately called the Cosine Circle of the quadrilateral, since the intercepts made by it on the sides $I B C, C D, D A, A B$ are $2 \rho \cos B A C, 2 \rho \cos C B D, 2 \rho \cos A C D, 2 \rho \cos A D B$ respectively.

When $\lambda=\frac{1}{2}, U$ is the mid-point of $O K$ and therefore coincident with the Brocard centre. The Tucker Circle having this point as its centre corresponds to the Lemoine or Triplicate Ratio Circle of the triangle. If $\tau^{\prime}$ be its radius, then putting $\lambda=\frac{1}{2}$ in (19), we have

$$
\tau^{\prime}=\frac{1}{2} R \sec \omega .
$$

The intercepts made on the sides are not, however, directly proportional to the simple cubes of the sides, for

$$
\begin{aligned}
H^{\prime} F & =u_{a}(\cot B+\cot C)=\frac{1}{2} a(\cot B+\cot C) \tan \omega \\
H^{\prime} F^{\prime} & =a-E E^{\prime}=a-E K-E^{\prime} K^{\prime}=a-u_{d} \operatorname{cosec} B-u_{b} \operatorname{cosec} C^{\prime} \\
& =a-\frac{1}{2}(d \operatorname{cosec} B+b \operatorname{cosec} C) \tan \omega \\
& =a\left(1-\frac{c d}{a b+c d}-\frac{b c}{a d+b c}\right), \text { from }(9) \\
& =\frac{a\left(a^{\prime \prime}-c^{2}\right)}{\triangle a^{2}} \text { from (4). }
\end{aligned}
$$$$
\text { or }
$$ 
Hence, $\quad H^{\prime}=\frac{1}{2} a(\cot B+\cot C) . \tan \omega=\frac{a\left(a^{2}-c^{2}\right)}{\Sigma a^{2}}$,

Similarly,

$$
\begin{aligned}
& E^{\prime} G=\frac{1}{2} b(\cot C+\cot D) \cdot \tan \omega=\frac{b\left(d^{2}-b^{2}\right)}{\Sigma a^{2}}, \\
& H F^{\prime \prime}=\frac{1}{2} c(\cot B+\cot C) \cdot \tan \omega=\frac{c\left(a^{2}-c^{2}\right)}{\Sigma a^{2}}, \\
& E G^{\prime}=\frac{1}{2} d(\cot C+\cot D) \cdot \tan \omega=\frac{d\left(d^{2}-b^{2}\right)}{\Sigma a^{2}}, \\
& \therefore \quad \begin{aligned}
H^{\prime} F: E^{\prime} G: H F^{\prime}: E G^{\prime} & =a^{3}-a c^{2}: l d^{3}-b^{3}: a^{2} c-c^{3}: d^{3}-b^{2} d \\
& =a^{3}-b c d: a c d-b^{3}: a b d-c^{3}: d^{3}-a b c .
\end{aligned}
\end{aligned}
$$

When $\lambda=0, U$ is coincident with $O$ and $\tau=R$; the Tucker Circle thus becomes the circumcircle.

\section{Ex-Cosine Circles.}

Let the tangents to the circumcircle drawn at $A, B, C, D$ intersect at $K_{1}, K_{2}, K_{3}, K_{4}$ respectively.

Through $K_{1}$ draw $K_{1} F_{1}$ parallel to $E^{\prime} F^{\prime}$ to meet $C B^{\prime}$ produced in $F_{1}$, and $K_{1} F_{1}^{\prime}$ parallel to $G F^{\prime}$ to meet $D A$ produced in $F_{1}^{\prime}$, then

$$
\angle K, F_{1} B=\angle E^{\prime} F C=\angle B D C=\angle B A C=\angle B H^{\prime} E=\angle K_{1} B F,
$$

since $K_{1} B \| E H^{\prime}$, both being perpendicular to $O B$.

$$
\therefore \quad K_{1} H_{1}^{\prime}=K_{1} B \text {. }
$$

Similarly,

$$
K_{1} F_{1}^{\prime}=K_{1} A \text {. }
$$

But

$$
\begin{aligned}
\angle K_{1} A O=\angle K_{1} B O & =\frac{1}{2} \pi, \text { and } O A=O B, \\
\therefore \quad K_{1} A & =K_{1} B,
\end{aligned}
$$

hence,

$$
K_{1} A=K_{1} B=K_{1} F_{1}=K_{1}^{\prime} F_{1}^{\prime} .
$$

$\therefore A, B, F_{1}^{\prime}, F_{1}^{\prime}$ lie on a circle whose centre is $K_{1}$.

Let $\rho_{1}=$ radius of this circle, then

$$
d=A B=2 \rho_{1} \cos K_{1} A B=2 \rho_{1} \cos A G^{\prime} H=2 \rho_{1} \cos A D B .
$$

This circle is thus an ex-cosine circle of the quadrilateral.

$$
\text { Again, } \quad \begin{aligned}
2 \rho_{1} \cos A D B=d & =2 R \sin A D B, \\
\therefore \quad \rho_{2}=R \tan A D B & =R \tan A C B
\end{aligned}
$$


Similarly, it may be shown that there are three other ex-cosine circles having $K_{2}, K_{3}, K_{4}$ as their centres. Let $\rho_{2}, \rho_{3}, \rho_{4}$ be their radii, then

$$
\left.\begin{array}{l}
\rho_{1}^{2}=R^{2} \tan ^{2} A C B=\frac{R^{2} d^{2}}{4 R^{2}-d^{2}} \\
\rho_{2}^{2}=R^{2} \tan ^{2} B D C=\frac{R^{2} a^{2}}{4 R^{2}-a^{2}} \\
\rho_{3}^{2}=R^{2} \tan ^{2} C A D=\frac{R^{2} b^{2}}{4 R^{2}-b^{2}} \\
\rho_{4}^{2}=R^{2} \tan ^{2} A B D=\frac{R^{2} c^{2}}{4 R^{2}-c^{2}}
\end{array}\right\}
$$

There are, however, two other ex-cosine circles having the diagonals as their respective chords. Let the tangents at $B, D$ intersect at $K_{e}$; through $K_{e}$ draw $H_{e} G_{e}^{\prime}$ parallel to $H G^{\prime}$ to meet $D A, B A$ produced in $H_{\theta}, G_{e}^{\prime}$, and draw $E_{e}^{\prime} F_{c}$ parallel to $E^{\prime} F^{\prime}$ to meet $C D, C B$ produced in $E_{c}^{\prime}, F_{e}$. Then it may readily be shown that $K_{c} B=K_{e} F_{e}=K_{c} H_{c}=K_{e} E_{e}^{\prime}=K_{e} G_{e}^{\prime}=K_{e} D$. Hence, $B, F_{e}, H_{e}, E_{e}^{\prime}, G_{c}^{\prime}, D$ lie on a circle whose centre is $K_{a}$.

Let $\rho_{e}$ be its radius, then

But

$$
\begin{aligned}
& e=B D=2 \rho_{e} \cos K_{e} B D=2 \rho_{e} \cos C . \\
& e=2 R \sin C . \\
\therefore \quad & \rho_{e}=R \tan C .
\end{aligned}
$$

Similarly it may be shown that there is another circle whose centre $K_{f}$ is the point of intersection of the tangents at $A$ and $C$ : if $\rho_{f}$ is its radius, then

$$
\rho_{e}=R \tan C, \rho_{f}=R \tan B .
$$

There are thus six ex-cosine circles to the quadrilateral.

The points $K_{e}, K_{f}$ bear also another significance, since $F_{e} E_{0}$ is parallel to $F E^{\prime}$ and is bisected at $K_{\bullet}$.

$\therefore C A$ and $A K$, are collinear.

Now

$$
A T_{1}: A K=O U: O K=C T_{3}: C K \text {. }
$$$$
\therefore A T_{1}: C T_{3}=A K: C K \text {. }
$$ 
Again, $\quad T_{1} H: K_{c} G_{c}^{\prime}=A T_{1}: A K_{\text {。 }}$

and $\quad T_{3} E^{\prime}: K_{\mathrm{e}} E_{c}{ }^{\prime}=C T_{3}: C K_{\mathrm{c}}$.

But $\quad T_{1}^{\prime} H=T_{3} E^{\prime}$ (Art. 14), and $K_{c} G_{e}^{\prime}=K_{c} E_{e}^{\prime}$.

$\therefore$ By division $A T_{1}: A K_{\mathrm{e}}=C T_{2}: C K_{\mathrm{e}}$.

or

$$
\begin{aligned}
A K_{\mathrm{r}}: C K_{e}=A T_{1}: C T_{3} \\
=A K: C K, \quad \text { from above, } \\
\quad \text { neglecting signs. }
\end{aligned}
$$

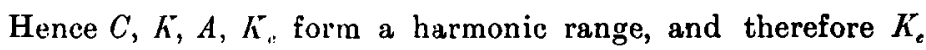
lies on the diagonal joining the intersection of $B A, C D$ produced with that of $C B, D A$ produced. Similarly, $K_{f}$ is the intersecting point of the diagonal through $K_{e}$ and $B D$ produced; thus the $\triangle K K_{c} K_{f}$ is the diagonal triangle of the complete quadrilateral formed by the four straight lines $A B, B C, C D, D A$.

18. Some relations between radii.

From $(20), \quad \tau^{2}=\frac{1}{4} R^{2} \sec ^{2} \omega$

$$
\begin{aligned}
& =\frac{1}{4} R^{2}\left(1+\tan ^{2} \omega\right) \\
& =\frac{1}{4}\left(R^{2}+\rho^{2}\right), \text { from }(18) .
\end{aligned}
$$

From (16), $\quad \beta^{2}=\frac{1}{4} R^{2} \sec ^{2} \omega \cdot \cos 2 \omega$

$$
\begin{aligned}
& =\frac{1}{4} R^{2}\left(2-\sec ^{2} \omega\right) \\
& =\frac{1}{4} R^{2}\left(1-\tan ^{2} \omega\right) \\
& =\frac{1}{4}\left(R^{2}-\rho^{2}\right), \text { from }(18) .
\end{aligned}
$$

Subtracting (24) from (23), we have

$$
\tau^{\prime 2}=\frac{1}{2} \rho^{2}+\beta^{2} \text {. }
$$

From $(15 a), \cot ^{2} \omega=\frac{\left(\sum a^{2}\right)^{2}}{16 Q^{2}}$,

and from (18),

$$
\begin{aligned}
\rho^{2}\left(\Sigma a^{2}\right)^{2} & =16 R^{2} Q^{2} \\
& =e^{2}(a b+c d)^{2}, \quad \text { from }(7), \\
& =2 a c(a d+b c)(a b+c d), \quad \text { from }(8), \\
& =2 a^{2} c^{2} . \Sigma a^{2}, \quad \text { from }(4) \\
\therefore \quad \rho^{2} . \Sigma^{2} & =2 a^{2} c^{2}=2 b^{2} d^{2}=2 a b c d . \quad \ldots \ldots \ldots \ldots \ldots \ldots \ldots
\end{aligned}
$$


From Fig. 3 we have

$$
\begin{aligned}
a & =u_{a}(\cot D B C+\cot A C B) . \\
\therefore \quad \cot \omega & =\frac{1}{2}(\cot D B C+\cot A C B) .
\end{aligned}
$$

But from (21), $R\left(\frac{1}{\rho_{1}}+\frac{1}{\rho_{3}}\right)=\cot A C B+\cot C A D$

$$
\begin{aligned}
& =\cot A C B+\cot D B C \\
& =2 \cot \omega, \\
& =\frac{2 R}{\rho}, \text { from }(18), \\
\therefore \quad \frac{1}{\rho_{1}}+\frac{1}{\rho_{3}} & =\frac{2}{\rho} .
\end{aligned}
$$

Similarly, $\frac{1}{\rho_{2}}+\frac{1}{\rho_{4}}=\frac{2}{\rho}$.

$$
\therefore \frac{1}{\rho_{1}}+\frac{1}{\rho_{3}}=\frac{1}{\rho_{2}}+\frac{1}{\rho_{4}}=\frac{2}{\rho} .
$$

Again, from (22),

$$
\begin{aligned}
R^{2}\left(\frac{1}{\rho_{e}^{2}}+\frac{1}{\rho_{f}^{2}}\right) & =\cot ^{2} C+\cot ^{2} B \\
& =\cot ^{2} A+\cot ^{2} B, \text { for } \cot C=-\cot A \\
& =\cot ^{2} \omega-1, \text { from }(15 c), \\
& =\frac{R^{2}}{\rho^{2}}-1 \\
& =\frac{R^{2}-\rho^{2}}{\rho^{2}}=\frac{4 \beta^{2}}{\rho^{2}}, \quad \text { from }(24), \\
\therefore \frac{1}{\rho_{e}^{2}}+\frac{1}{\rho_{f}^{2}} & \left.=\frac{1}{\rho^{2}}-\frac{1}{R^{2}}=\frac{4 \beta^{2}}{R^{2} \rho^{2}} . \ldots \ldots \ldots \ldots \ldots \ldots(28)\right)
\end{aligned}
$$

19. Comparison of Formulae.

To summarise the analogy, the principal formulae for both the triangle and the cyclic quadrilateral are here collected and placed side by side. 
TRIANCI.E.

$X X^{\prime}=2 R \sin \omega \cdot(2 \cos$
$\omega<30^{\circ}$
$\cot \omega=\Sigma \cot \Lambda=\frac{\Sigma a^{2}}{4 \triangle}$

$\operatorname{cosec}^{2}(1)=\frac{\Sigma a^{2} b^{2}}{4 \triangle^{2}}=\Sigma \operatorname{cosec}^{2} A$

$\cos ^{2}()=\frac{\left(\Sigma a^{2}\right)^{2}}{4 \operatorname{La}^{2} b^{2}}$

$\sin 2 \omega=\frac{2 \Delta \cdot \Sigma a^{2}}{\triangle a^{2} b^{2}}$

$\cos 2 \omega=\frac{\Sigma a^{4}}{2 \Sigma a^{2} b^{2}}$

$\beta=\frac{1}{2} R \sec \omega \cdot(2 \cos 2 \omega-1)^{\frac{1}{2}}$

$\rho=R \tan \omega$

$\tau^{2}=R^{2}\left(1-2 \lambda+\lambda^{2} \sec ^{2} \omega\right)$

$\rho_{\mathrm{a}}=R \tan A$

$\tau^{\prime 2}=\frac{1}{4}\left(R^{2}+\rho^{2}\right)=\frac{1}{4} R^{2} \sec ^{2} \omega$

$\beta^{2}=\frac{1}{4}\left(R^{2}-3 \rho^{2}\right)=\tau^{\prime 2}-\rho^{2}$

$\Sigma \frac{1}{\rho_{a}}=\frac{1}{\rho}$.
CYCLIC QUADRILATERAL

$$
a c=b d .
$$

$X X^{\prime} \quad=2 R \sin \omega \cdot \cos ^{\frac{1}{2}} 2 \omega$

$\omega<45^{\circ}$

$\cot ^{2} \omega=1+\cot ^{2} A+\cot ^{2} B$

$\cot \omega=\frac{\Sigma a^{2}}{4 Q}$

$\operatorname{cosec}^{2} \omega=\frac{\Sigma a^{2} b^{2}+2 a^{2} c^{2}}{4 Q^{2}}$

$=\operatorname{cosec}^{2} A+\operatorname{cosec}^{2} B$

$\cos ^{2} \omega=\frac{\left(\sum a^{2}\right)^{2}}{4\left(\Sigma^{2} a^{2} b^{2}+2 a^{2} c^{2}\right)}$

$\sin 2 \omega=\frac{2 Q \cdot \dot{2} a^{2}}{\Sigma a^{\prime \prime} b^{2}+2 a^{2} c^{2}}$

$\cos 2 \omega=\frac{\Sigma a^{4}-4 a^{2} c^{2}}{2\left(\Sigma a^{2} b^{2}+2 a^{2} c^{2}\right)}$

$\beta=\frac{1}{2} R \sec \omega \cdot \cos ^{\frac{1}{2}} 2 \omega$

$\rho=R \tan \omega$

$\tau^{2}=R^{2}\left(1-2 \lambda+\lambda^{2} \sec ^{2} \omega\right)$

$\rho_{\varepsilon}=R \tan C$

$\tau^{\prime 2} \quad=\frac{1}{4}\left(R^{2}+\rho^{2}\right)=\frac{1}{4} R^{2} \sec ^{2} \omega$

$\beta^{2}=\frac{1}{4}\left(R^{2}-\rho^{2}\right)=\tau^{\prime 2}-\frac{1}{2} \rho^{2}$

$\sum \frac{1}{\rho_{1}}=\frac{4}{\rho}$. 